\title{
Considerations for clinical trials to study cannabinoids in Canadian children with neurologic disorders
}

\author{
Lauren E. Kelly PhD, Anne Junker MD
}

Cite as: CMAJ 2018 August 27;190:E1002-3. doi: 10.1503/cmaj.180605

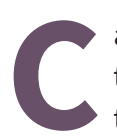

anadian parents are increasingly turning to cannabinoids to treat children with neurologic disorders. ${ }^{1}$ The evidence base to support this choice consists of little more than a small series of case reports, but many parents are guided by anecdotal online reviews in parenting communities. ${ }^{2-5}$ Without licensed products supported by high-quality evidence, unregulated use of cannabinoids in children will continue, particularly given the impending legalization of cannabis in Canada and parents' desperation for effective treatments. There is an urgent need for rigorous clinical trials to research the use of cannabinoids in pediatric patients. It is time to move away from the "era of the anecdote" and toward evidenceinformed selection and dosing of cannabinoids in children. ${ }^{6,7}$

Parents use cannabinoids to manage seizures, spasticity and irritability in children with epilepsy, autism-spectrum disorder and cerebral palsy. ${ }^{8}$ Anecdotally, many acquire products for their children from a variety of sources, including online. ${ }^{3,5}$ The use of cannabinoids to treat some pediatric conditions has become so common that recruiting cannabinoid-naive children for clinical trials has become challenging. For example, upward of $90 \%$ of children with aggressive brain tumours are being treated with high-tetrahydrocannabinol cannabinoids by their parents, who are accessing these drugs independently of care providers (Dr. Rod Rassekh, personal communication, 2018).

However, some research has been conducted in this area. In June 2018, the US Food and Drug Administration approved Epidiolex, an oral cannabidiol formulation, for the treatment of Dravet syndrome and Lennox-Gastaut syndrome. A placebo-controlled trial in children aged 2 to 18 years found that cannabidiol reduced seizure frequency but was associated with adverse events, including somnolence and elevated liver-enzyme levels, when added to standard care. ${ }^{9}$

Overall, though, given uncertainty regarding the effectiveness of cannabinoids, health care providers have an ethical imperative to enrol patients in randomized placebo-controlled clinical trials the gold standard for developing evidence on safety and efficacy. It is unclear how many of the companies that have applied to Health Canada to become licensed medical producers of cannabis will be developing pediatric products. The two small pediatric dose-finding trials that are ongoing in Canada are examining the effectiveness of two different products: CanniMed 1:20 $(1.0 \mathrm{mg} / \mathrm{mL}$ tetrahydrocannabinol, $20 \mathrm{mg} / \mathrm{mL}$ cannabidiol) and TIL-TC150. To

\section{KEY POINTS}

- Canadian parents are using unregulated cannabinoids for their children with neurologic conditions, and many are obtaining these products online.

- Clinical trials are needed to determine the safety and effectiveness of cannabinoids and to guide dosing in children with complex neurological conditions.

- Unique clinical trial design considerations will require prospective planning, innovative methods, parent engagement and multisite collaboration.

guide the development of more clinical trials of cannabinoids in children with neurologic disorders, several aspects of study design must be considered.

Because conditions for which cannabinoids may be indicated in children are rare, multisite studies will be required. The investigation of many products for the same condition hinders recruitment efforts, as studies compete for participants across sites. National collaborations involving academic, industry and provincial governments would be prudent, given the substantial investment required for multisite recruitment and follow-up. Ideally, a prospective plan on how best to recruit and retain participants across multiple sites should be devised, involving parent groups. Long-term follow-up is also important, but it adds the challenges of evolving assent and consent models as children age and transition to adult services. Given that the effects of cannabinoids may be dependent on the age of exposure, recruitment should be stratified by age group. ${ }^{1}$ Because the potential long-term harms related to medicinal use of cannabinoids are unclear, consent processes should be iterative and updated as new evidence emerges.

There is limited evidence regarding the optimal dose, strain and ratio of cannabidiol to tetrahydrocannabinol. ${ }^{10}$ Although both tetrahydrocannabinol and cannabidiol are neuroactive, tetrahydrocannabinol produces most of the psychoactive effects. ${ }^{11}$ Development of age-appropriate formulations must consider that the lipophilic nature of cannabinoids may affect weight-based dosing. Child-friendly delivery methods, such as buccal spray or oil, with controlled doses, would 
be appropriate. Pesticides and heavy-metal contaminants present concerns in unregulated products. As a regulatory requirement, the production of cannabinoid agents should comply with Good Manufacturing Practices to ensure consistency and quality.

A lack of previous evidence in this area presents challenges for determining traditional clinical trial operational characteristics, such as expected variability or effect size. Limited data exist on expected cannabinoid response variability in children ${ }^{7}$ and, without a clear effectiveness outcome, sample-size calculations become estimates at best. A "no treatment" or placebo control arm is unacceptable for most neurologic disorders, given the risk of harm of no treatment. Detecting a treatment difference with an active control requires larger sample sizes, to allow for a trial to be adequately powered. Given impending cannabis legalization in Canada, contamination of control groups will be a concern. Strict protocols for blinding participants should be designed to prevent parents from seeking adjuvant cannabinoid products online. ${ }^{4,6}$

Pediatric neurologic disorders are heterogeneous. This creates variability in the level of risk acceptable to parents who are looking to find an effective treatment. Parents of children with less severe illnesses may be less likely to consent to cannabinoid studies, which may affect recruitment and the ability to power a trial. Flexible and innovative study designs - such as outcome-adaptive randomization, crossover studies or a platform trial - may present options for improved trial efficiency and increased speed. This would allow for answers to important research questions to be obtained ethically and to regulatory standards.

It is important to define what constitutes condition-specific meaningful improvement, and this can be achieved only through engagement with families. Close monitoring of drug-drug interactions and adverse reactions previously associated with cannabinoids - such as nausea, somnolence, weakness, mood changes, anxiety, dependence and suicide risk - is essential within pediatric trials. Withdrawal syndromes have been reported after cannabinoid exposure is stopped in adults. ${ }^{12}$ Trial designs should include management plans for potential withdrawal syndromes. Long-term developmental assessments should include neuroimaging. ${ }^{1}$

There is a paucity of evidence surrounding biomarkers as surrogate measures for long-term neurodevelopmental outcomes. Objective measures, such as electroencephalography for seizures, electromyography for spasticity or video monitoring of autistic behaviours, may be valuable to evaluate effectiveness objectively, given that trial participation itself may influence subjective outcomes. As cannabinoid policies vary across regions, formal documentation of medical exemption should be provided to participants who wish to travel during the study period. Furthermore, there is an ethical imperative to plan prospectively for participants to receive the investigation product upon study completion if desired. This is critical to ensure that, if benefit is perceived, desperate parents do not resort to seeking unregulated cannabinoid products once access to the study drug ends.

Although trials of cannabinoid treatments in children with neurologic disorders certainly qualify as high-risk research in a vulnerable population, excluding this population from high-quality research may deny these patients access to safe and evidence-based treatments. Physicians, understandably, lack confidence in prescribing cannabinoids and in supporting parents who are self-prescribing cannabinoids, and require an evidence base to guide them. Strong evidence on the risk-benefit profile for cannabis treatments is lacking for children, which increases the challenge of informing parents of potential risks. ${ }^{8,10}$ Epidemiologic data from recreational use studies do not represent robust evidence because dosing may be intermittent, resulting in variably sustained cannabinoid levels, and different routes of administration affect cannabinoid pharmacokinetics.

The medical community, regulators, patients and families must apply the same standards to cannabinoids as would be required for other therapeutic agents used to treat pediatric conditions. Strong parental and physician advocacy is needed to ensure that cannabinoids are adequately and safely researched for children with neurologic disorders in Canada.

\section{References}

1. Rieder MJ. Is the medical use of cannabis a therapeutic option for children? Paediatr Child Health 2016;21:31-4.

2. Maa E, Figi P. The case for medical marijuana in epilepsy. Epilepsia 2014;55:783-6.

3. Porter BE, Jacobson C. Report of a parent survey of cannabidiol-enriched cannabis use in pediatric treatment-resistant epilepsy. Epilepsy Behav 2013;29:574-7.

4. Hussain SA, Zhou R, Jacobson C, et al. Perceived efficacy of cannabidiol-enriched cannabis extracts for treatment of pediatric epilepsy: a potential role for infantile spasms and Lennox-Gastaut syndrome. Epilepsy Behav 2015;47:138-41.

5. Press CA, Knupp KG, Chapman KE. Parental reporting of response to oral cannabis extracts for treatment of refractory epilepsy. Epilepsy Behav 2015;45:49-52.

6. Hadland SE, Knight JR, Harris SK. Medical marijuana: review of the science and implications for developmental behavioral pediatric practice. J Dev Behav Pediatr 2015;36:115-23.

7. Wong SS, Wilens TE. Medical cannabinoids in children and adolescents: a systematic review. Pediatrics 2017;140:e20171818.

8. Huntsman RJ, Tang-Wai R, Acton B, et al. Cannabis for the treatment of paediatric epilepsy? An update for Canadian paediatricians. Paediatr Child Health Available: pxy036, https://doi.org/10.1093/pch/pxy036 (accessed 2018 April 28).

9. Devinsky O, Cross JH, Laux L, et al. Trial of cannabidiol for drug-resistant seizures in the Dravet syndrome. N Engl J Med 2017;376:2011-20.

10. Whiting PF, Wolff RF, Deshpande S, et al. Cannabinoids for medical use: a systematic review and meta-analysis. JAMA 2015;313:2456-73.

11. Devinsky O, Cilio MR, Cross H, et al. Cannabidiol: pharmacology and potential therapeutic role in epilepsy and other neuropsychiatric disorders. Epilepsia 2014; 55:791-802.

12. Budney AJ, Hughes JR. The cannabis withdrawal syndrome. Curr Opin Psychiatry 2006;19:233-8.

\section{Competing interests: None declared.}

This article has been peer reviewed.

Affiliations: Department of Pediatrics and Child Health (Kelly), Rady College of Medicine, University of Manitoba, Winnipeg, Man.; Clinical Trials Platform (Kelly), George and Fay Yee Centre for Healthcare Innovation, Winnipeg, Man.; Division of Allergy and Immunology
(Junker), British Columbia Children's Hospital, Vancouver, BC; Department of Pediatrics, Faculty of Medicine (Junker), University of British Columbia, Vancouver, BC

Contributors: Anne Junker contributed to the conception of the work. Lauren Kelly designed the work. Both authors drafted the manuscript, revised it critically for important intellectual content, gave final approval of the version to be published and agreed to be accountable for all aspects of the work.

Acknowledgements: The authors would like to acknowledge Drs. Timothy Oberlander (MD) and Thierry Lacaze (MD PhD) for their contributions and thoughtful review of this commentary.

Correspondence to: Lauren Kelly, Lauren.Kelly@umanitoba.ca 